\title{
Weakly Nonlinear Analysis of Vortex Formation in a Dissipative Variant of the Gross-Pitaevskii Equation
}

\author{
J.C. Tzou* P.G. Kevrekidisł T. Kolokolnikov
}

September 10, 2018

\begin{abstract}
For a dissipative variant of the two-dimensional Gross-Pitaevskii equation with a parabolic trap under rotation, we study a symmetry breaking process that leads to the formation of vortices. The first symmetry breaking leads to the formation of many small vortices distributed uniformly near the Thomas-Fermi radius. The instability occurs as a result of a linear instability of a vortex-free steady state as the rotation is increased above a critical threshold. We focus on the second subsequent symmetry breaking, which occurs in the weakly nonlinear regime. At slightly above threshold, we derive a onedimensional amplitude equation that describes the slow evolution of the envelope of the initial instability. We show that the mechanism responsible for initiating vortex formation is a modulational instability of the amplitude equation. We also illustrate the role of dissipation in the symmetry breaking process. All analyses are confirmed by detailed numerical computations.
\end{abstract}

Keywords: Nonlinear Schrödinger equation, Bose-Einstein condensates, Vortex nucleation, Dissipative Gross-Pitaevskii equation.

AMS subject classifications: 35Q55, 76M23, 76A25.

\section{Introduction}

The topic of vortex formation upon rotation of an atomic Bose-Einstein condensate has received a tremendous volume of attention during the past 15 years, with many of the relevant results finding their way in main archival references on the subject, including the books [27, 30. This is natural, not only because of the inherent interest in vortices as fundamental structures in this and more generally in atomic, quantum, and superfluids systems [28, but also because this has been a prototypical way of introducing vortices in the system. These studies not only include theoretical works but also numerous experiments, in isotropic and anisotropic settings, with few or with many atoms, in oblate or prolate traps in at least four distinct experimental groups pioneering the early experiments [4, 16, 22, 17. Even far more recent experiments, relying chiefly on other techniques, including the Kibble-Zurek mechanisms utilize rotation as a way of controllably producing vortices of a given (same) charge [25].

It is then natural to expect a large volume of theoretical literature tackling the relevant theme. It was realized early on that the surface excitations play a crucial role in the relevant "instability" that leads to the emergence of vortices [9. The work of Isoshima and Machida [18] was among the first that recognized the complex energetic balance between the different scenarios (e.g. stable, metastable or potentially unstable nonvortex states, and similarly for vortex bearing states). This metastability opens the potential for hysteretic

\footnotetext{
*Department of Mathematics and Statistics, Dalhousie University, Halifax, Canada

${ }^{\dagger}$ Department of Mathematics and Statistics, University of Massachusetts, Amherst, MA 01003-4515, USA; Center for Nonlinear Studies and Theoretical Division, Los Alamos National Laboratory, Los Alamos, NM 87544 USA

${ }^{\ddagger}$ Department of Mathematics and Statistics, Dalhousie University, Halifax, Canada

$\S$ Nonlinear Dynamical System Group, (URL: http://nlds.sdsu.edu), Computational Science Research Center, (URL: http://www.csrc.sdsu.edu), and Department of Mathematics and Statistics, San Diego State University, San Diego, California 92182-7720, USA
} 
phenomena, depending on whether, in accordance with the experiment, the rotation frequency was ramped up or ramped down, as illustrated, e.g., in Ref. [14]. Numerous simulations also followed these earlier works, including, at different levels, finite temperature considerations. More specifically, both Refs. [26], as well as Ref. 39] considered the finite temperature model of the so-called dissipative Gross-Pitaevskii equation (see details below), and of ramps therein, as a prototypical system where the nucleation and emergence of vortex lattices was spontaneous. On the other hand, the work of Ref. 37] used the framework of the Hartree-FockBogoliubov method (in the so-called Popov approximation) as a means of self-consistently including thermal effects, finding that the particular value of the temperature may affect the number of vortices formed.

From a theoretical perspective, there have been, to the best of our understanding, two distinct schools of thought. One of these, based on the work of Ref. [5] (see also importantly the later interpretation of Ref. [10], for the case of a toroidal trap), is based on computing the Landau criterion threshold, i.e., identifying the order of the mode that will be associated with the Landau instability and inferring from that the number of vortices that will emerge. A distinct approach pioneered by the work of Stringari and collaborators [34, 21] (see also Ref. [38, as well as the review of the relevant considerations in Ref. [30]) involved the bifurcation - from the ground state, be it isotropic or anisotropic - of additional states, beyond the rotation frequency that renders neutral (i.e., of vanishing frequency) the quadrupolar mode. These two approaches have both been developed in the limit of large chemical potential, yet to the best of our knowledge, they have never quite been "reconciled" with each other, aside from a short remark in the work of Ref. [5] suggesting that Landau method is more relevant when surface excitations are crucial, while if the instability has a more global character (e.g., for smaller atom numbers), then the hydrodynamic approach of Refs. 30, 34, 21, 38, is more suitable.

While understanding these two approaches, their similarities and differences, and providing a unified perspective of this problem based on them appears to us an intriguing problem for further study, we will not pursue it further here. Instead, we will focus on characterizing exactly how a vortex is "born" and migrates inwards towards the center of the trap. We will build on our earlier work 8 ] where we used a multi-scale expansion to obtain a reduction of the relevant eigenvalue problem, associated with the vortex forming instability. In our case, where the model of choice is the dissipative Gross-Pitaevskii equation (DGPE), we argued that there is a true instability, contrary to what is the case with the Hamiltonian case, where the eigenvalues simply cross the origin of the spectral plane changing "energy" or "signature" — see the details of Ref. [8. Here, we take this analysis a significant step further, by reducing the relevant dynamics, at the periphery of the atomic cloud, to an effective one-dimensional azimuthal strip.

Remarkably, we find that although the original dynamics pertains to a self-defocusing Gross-Pitaevskii equation (GPE), this reduced azimuthal evolution bears a self-focusing character. This trait is manifested through the emergence of a modulational instability (MI) against the backdrop of the homogeneous background. This, in turn, results in a "spike" emerging as subtracted from the background, which finally will morph into a vortex initially rotating along the strip and gradually spiraling inwards in accordance with its dynamical equation of motion — for vortex motion within the DGPE realm see, e.g., Ref. [41] ${ }^{1}$. Our emphasis here will be in highlighting the mechanism leading to the vortex formation, offering quantitative comparisons of our focusing GPE reduction (and its MI mechanism) with the full two-dimensional numerical results. Our presentation is structured as follows. In Sec. 2, we briefly present the mathematical setup. In Sec. 3, we discuss the weakly nonlinear analysis and the derivation of the effective one-dimensional self-focusing GPE. In Sec. 4, we analyze the MI and compare its predictions to the full system. Finally in Sec. 5, we summarize our findings and present some directions for future study.

\section{Mathematical Setup}

Our starting point will be the dissipative variant of the Gross-Pitaevskii equation (DGPE) of the form [29] (see also the more recent works of Refs. [26, 39])

$$
(\gamma-i) u_{t}=\frac{1}{2} \Delta u+\left(\mu-\frac{1}{2} \Omega_{\text {trap }}^{2} \rho^{2}\right) u-|u|^{2} u-i \Omega_{\mathrm{rot}} u_{\theta},
$$

\footnotetext{
${ }^{1}$ In the latter case, the precession corresponds to an anomalous (negative energy or signature) mode and hence the spiraling occurs outwards, but in the presence of rotation this mode becomes normal and similar dynamical equations describe the spiraling inwards.
} 
where $|u(\rho, \theta, t)|^{2}$ is the time-dependent two-dimensional density of the atomic condensate cloud within a parabolic trap of strength $\Omega_{\text {trap }}$ and $\gamma$ accounts for a phenomenological temperature-dependent dissipation effect (see, e.g., Refs. 31, 26, 32, 17, 19, 15]). This phenomenological dissipation term provides a prototypical way of effectively accounting for the interaction of the condensate with the thermal cloud. Physically relevant values of $\gamma>0$ are of magnitude $1 \times 10^{-3}$; see, e.g., Ref. 41]. Equation (1) is already written in the corotating frame of the trap rotating with frequency $\Omega_{\text {rot }}$. The chemical potential $\mu$ is a measure of the strength of interaction between atoms, which we assume to be large in comparison to all other parameters in Eq. (1). This assumption motivates the following scaling and definitions

$$
t=\frac{1}{\mu} T, \quad \rho=\frac{1}{\Omega_{\text {trap }}} \sqrt{2 \mu} r, \quad u(\rho, \theta, t)=\sqrt{\mu} W(r, \theta, T) ;
$$

where

$$
\widetilde{\Omega} \equiv \frac{1}{\mu} \Omega_{\mathrm{rot}}, \quad \varepsilon \equiv \frac{1}{2 \mu} \Omega_{\text {trap }} \ll 1,
$$

so that, in rescaled form, the DGPE may be written as

$$
(\gamma-i) W_{T}=\varepsilon^{2} \Delta W+\left(1-r^{2}\right) W-|W|^{2} W-i \widetilde{\Omega} W_{\theta} ; \quad \gamma>0,
$$

where the edge of the atomic cloud, the Thomas-Fermi radius, is now rescaled to $r=1$. A radially symmetric, vortex-free, steady state $W=W_{0}(r)$ of Eq. (2) exists and satisfies

$$
W_{0 r r}+\frac{1}{r} W_{0 r}+\left(1-r^{2}\right) W_{0}-\left|W_{0}\right|^{2} W_{0}=0, \quad\left|W_{0}\right| \rightarrow 0 \text { as } r \rightarrow \infty .
$$

Notice that the steady state profile is identical to the one of the corresponding Hamiltonian $(\gamma=0)$ model.

Let us use here an approach extending our recent considerations in Ref. [8]. In that work, for increasing rotation frequency $\widetilde{\Omega}$, it was observed that the steady state $W_{0}$ first loses stability to a spatial mode scaling as $\mathcal{O}\left(\varepsilon^{-2 / 3}\right)$. This instability manifests initially in a large number of small vortices distributed uniformly near the Thomas-Fermi radius $r=1$. In fact, the relevant surface mode going unstable is the one placing in the periphery of the system the number of vortices filling it by spanning their respective healing lengths. This behavior was analyzed in Ref. [8], showing it was due to a linear instability of $W_{0}$-within the DPGE setting, although the Hamiltonian solution was still identified as dynamically stable - with increasing rotation frequency. Numerical solutions of Eq. (2) revealed that a subsequent symmetry breaking mechanism causes only a fraction of these vortices to persist and be pulled into the bulk of the condensate. For our current considerations, for $\widetilde{\Omega}$ slightly above threshold, we perform a weakly nonlinear analysis to examine the onset of this second symmetry breaking process. While the analysis does not predict the fraction of vortices that survive or their eventual fate as they form in the fully nonlinear regime, our analysis accurately captures all of the dynamics in the early stages of their development. In particular, we show that the weakly nonlinear dynamics of the two-dimensional self-defocusing system (2) is described by a one-dimensional perturbed self-focusing nonlinear Schrödinger equation (NLSE). This is both perhaps intuitively unexpected and at the same time crucially relevant to the observed phenomenology. This is because, as our analysis shows, the initial pattern selection mechanism responsible for the formation of vortices is a MI of a non-stationary uniform solution of the one-dimensional amplitude equation, a mechanism (within the continuum, cubic nonlinearity considered herein) restricted to the self-focusing variant of the GPE problem.

\section{Weakly nonlinear analysis and amplitude equations}

Since vortices nucleate near the Thomas-Fermi $(r=1)$ radius with critical wavenumber $m \sim \varepsilon^{-2 / 3} m_{0}$ when $\widetilde{\Omega} \sim \varepsilon^{4 / 3} \Omega$ with $m_{0}, \Omega \sim \mathcal{O}(1)$, we rescale Eq. $(2)$ according to

$$
r=1+\varepsilon^{2 / 3} x, \quad \theta=\varepsilon^{2 / 3} y, \quad T=\varepsilon^{-2 / 3} t, \quad W=\varepsilon^{1 / 3} w, \quad \widetilde{\Omega}=\varepsilon^{4 / 3} \Omega .
$$

This way, we are restricting our consideration to the small strip of space near the Thomas-Fermi radius, while considering small amplitude solutions (since the density approaches zero near that limit), for longer 
time scales such that the vorticity is expected to emerge. In these rescaled variables, Eq. (2) to leading order becomes

$$
(\gamma-i) w_{t}=w_{x x}+w_{y y}-\left(2 x+|w|^{2}\right) w-i \Omega w_{y}
$$

with

$$
|w| \sim \sqrt{-2 x} \text { as } x \rightarrow-\infty \text {, and }|w| \rightarrow 0 \text { as } x \rightarrow \infty,
$$

where $w$ is periodic in $y$. In arriving at Eq. (3) from Eq. (2), the largest terms that have been dropped are of order $\mathcal{O}\left(\varepsilon^{2 / 3}\right)$. The focus of the analysis and computations herein will be on Eq. (3).

Writing $w=u+i v$ with $u, v \in \mathbb{R}$, we rewrite Eq. (3) as the system

$$
\begin{aligned}
& \gamma u_{t}+v_{t}=u_{x x}+v_{y y}-\left(2 x+u^{2}+v^{2}\right) u+\Omega v_{y}, \\
& \gamma v_{t}-u_{t}=v_{x x}+v_{y y}-\left(2 x+u^{2}+v^{2}\right) v-\Omega u_{y} .
\end{aligned}
$$

A steady state of Eqs. (4) may be written as $u=u_{0}(x)$ and $v=0$, where $u_{0}(x)$ is the unique solution of a Painlevé II equation

$$
u_{0}^{\prime \prime}=2 x u_{0}+u_{0}^{3},
$$

with limiting conditions (see, e.g., Ref. [1])

$$
u_{0} \sim \sqrt{-2 x} \text { as } x \rightarrow-\infty, \quad u_{0} \rightarrow 0 \text { as } x \rightarrow \infty .
$$

With respect to the full system (2), $u_{0}$ is the corner layer near $r=1$ of the steady state solution $W_{0}(r)$. Next, we let $u=u_{0}(x)+\phi(x, y, t)$ and $v=\psi(x, y, t)$ in Eq. (4) to obtain

$$
\begin{aligned}
\gamma \phi_{t}+\psi_{t} & =\phi_{x x}+\phi_{y y}-\left(2 x+3 u_{0}^{2}\right) \phi-3 u_{0} \phi^{2}-u_{0} \psi^{2}-\psi^{2} \phi-\phi^{3}+\Omega \psi_{y}, \\
\gamma \psi_{t}-\phi_{t} & =\psi_{x x}+\psi_{y y}-\left(2 x+u_{0}^{2}\right) \psi-2 u_{0} \phi \psi-\phi^{2} \psi-\psi^{3}-\Omega \phi_{y} .
\end{aligned}
$$

The steady state of Eq. (5) is then $\phi=\psi=0$. Assuming a perturbation of the form $(\phi, \psi)=(i A(x), B(x)) e^{i m y+\lambda t}$ in Eq. (5) (given the invariance of the solution along the angular variable and the periodicity of the latter, we decompose it in Fourier modes) and collecting linear terms, we obtain the eigenvalue problem

$$
\begin{aligned}
A^{\prime \prime}-m^{2} A-\left(2 x+3 u_{0}^{2}\right) A+m \Omega B_{1} & =\lambda(\gamma A+B), \\
B^{\prime \prime}-m^{2} B-\left(2 x+u_{0}^{2}\right) B+m \Omega A_{1} & =\lambda(\gamma B-A) .
\end{aligned}
$$

As in Ref. [8], we set $\lambda=0$ in Eq. (6) and solve the associated eigenvalue problem for $\Omega(m)$, yielding the neutral stability curve depicted in Fig. 1(a) (see Ref. 8] for a detailed analysis and full results). We denote $\Omega_{0}$ as the smallest value of $\Omega$ at which the steady state loses stability to a perturbation with critical wavenumber $m_{0}$ (see Fig. 1(a)p. Then, when $\Omega=\Omega_{0}+\delta^{2}$ with $\delta \ll 1$, numerical computations show that $\Re(\lambda) \sim \Im(\lambda) / \gamma \sim \mathcal{O}\left(\delta^{2}\right)$. This is depicted in Fig. 1(b).

To analyze the slow evolution of this perturbation slightly above threshold, we assume the asymptotic expansion

$$
\Omega=\Omega_{0}+\delta^{2} \Omega_{2} ; \quad \phi=\delta \phi_{1}+\delta^{2} \phi_{2}+\delta^{3} \phi_{3}, \quad \psi=\delta \psi_{1}+\delta^{2} \psi_{2}+\delta^{3} \psi_{3} ; \quad 0<\delta \ll 1 .
$$

The expansion in Eq. (7a) is motivated by the expectation that the bifurcation is of the pitchfork type, for which $\phi, \psi \sim \mathcal{O}\left(\sqrt{\Omega-\Omega_{0}}\right)$. The solvability condition is then expected to arise at $\mathcal{O}\left(\delta^{3}\right)$. Recalling that the lowest order term omitted from the leading order in Eq. (3) is of order $\mathcal{O}\left(\varepsilon^{2 / 3}\right)$, we require that $\delta^{3} \gg \varepsilon^{2 / 3}$. We next introduce the slow spatial and temporal scales

$$
x=X / \delta, \quad y=Y / \delta, \quad t=T / \delta^{2} .
$$

The spatial scale is motivated by the $\mathcal{O}(\delta)$ band of wavenumbers that acquires a positive growth rate as $\Omega$ is increased an $\mathcal{O}\left(\delta^{2}\right)$ distance above threshold (see Fig. 1(a)d, while the temporal scale is motivated by the corresponding scaling of $\lambda$ in Eq. (6) (see Fig. 1(b)]. Below, we assume that $\gamma=\mathcal{O}(1)$ with respect to $\delta$. 


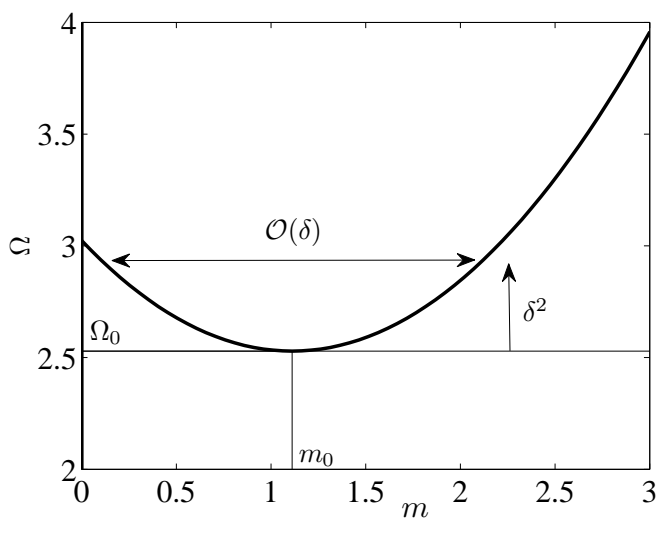

(a) spatial scale

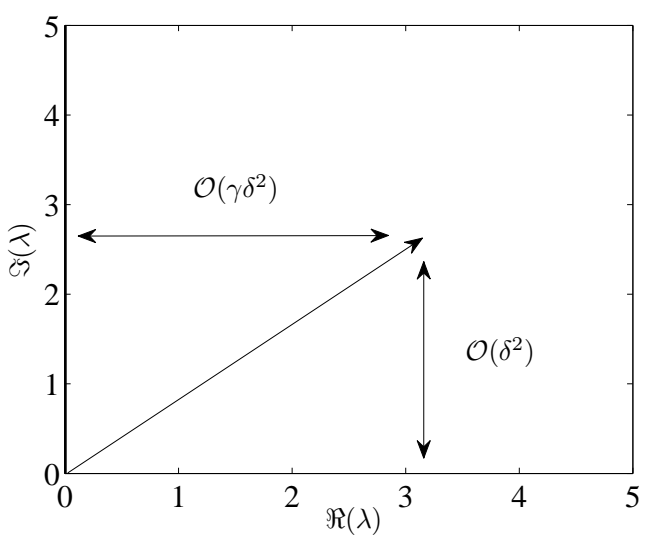

(b) eigenvalue scaling

Figure 1: (a) A depiction of the neutral stability curve $\Omega(m)$ (solid thick line). As $\Omega$ is increased by $\mathcal{O}\left(\delta^{2}\right)$ above $\Omega_{0}$, an $\mathcal{O}(\delta)$ band of wavenumbers around $m=m_{0}$ acquires positive growth rate. (b) The scalings of $\Re(\lambda)$ and $\Im(\lambda)$ are depicted as $\Omega$ is increased by $\mathcal{O}\left(\delta^{2}\right)$ above $\Omega_{0}$. The figures here are for illustrative purposes only. See Ref. [8] for full results and a detailed analysis.

Substituting Eq. (7) into Eq. (5), we solve the linear problems at successively higher orders of $\delta$. At $\mathcal{O}(\delta)$, we obtain the linear terms of Eq. (5)

$$
\begin{aligned}
\gamma \phi_{1 t}+\psi_{1 t} & =\phi_{1 x x}+\phi_{1 y y}-\left(2 x+3 u_{0}^{2}\right) \phi_{1}+\Omega_{0} \psi_{1 y} \\
\gamma \psi_{1 t}-\phi_{1 t} & =\psi_{1 x x}+\psi_{1 y y}-\left(2 x+u_{0}^{2}\right) \psi_{1}-\Omega_{0} \phi_{1 y} .
\end{aligned}
$$

We calculate a $t$-independent solution to Eq. (8) of the form

$$
\left(\begin{array}{c}
\phi_{1} \\
\psi_{1}
\end{array}\right)=C(X, Y, T)\left(\begin{array}{c}
i A_{1}(x) \\
B_{1}(x)
\end{array}\right) e^{i m_{0} y}+\text { c.c. },
$$

where c.c. denotes the complex conjugate. Here, $A_{1}(x)$ and $B_{1}(x)$ are real and satisfy

$$
L_{m_{0}}\left(\begin{array}{c}
A_{1} \\
B_{1}
\end{array}\right) \equiv\left(\begin{array}{c}
A_{1}^{\prime \prime}-m_{0}^{2} A_{1}-\left(2 x+3 u_{0}^{2}\right) A_{1}+m_{0} \Omega_{0} B_{1} \\
B_{1}^{\prime \prime}-m_{0}^{2} B_{1}-\left(2 x+u_{0}^{2}\right) B_{1}+m_{0} \Omega_{0} A_{1}
\end{array}\right)=0, \quad A_{1}, B_{1} \rightarrow 0 \text { as } x \rightarrow \pm \infty,
$$

with

$$
m_{0} \approx 1.111, \quad \Omega_{0} \approx 2.529 .
$$

In addition, we impose the normalization constraint

$$
\int_{-\infty}^{\infty} A_{1}^{2}+B_{1}^{2} d x=1 ; \quad A_{1}, B_{1}>0 .
$$

In Eq. (9), $C(X, Y, T)$ is a complex quantity that describes the slowly modulated envelope of the perturbation, while in Eq. $\left[10 \mathrm{~b}, m_{0}\right.$ is the critical wavenumber that first becomes unstable as $\Omega$ is increased above $\Omega_{0}$.

At $\mathcal{O}\left(\delta^{2}\right)$, we have

$$
\begin{aligned}
\phi_{2 x x}+\phi_{2 y y}-\left(2 x+3 u_{0}^{2}\right) \phi_{2}+\Omega_{0} \psi_{2 y} & =3 u_{0} \phi_{1}^{2}+u_{0} \psi_{1}^{2}-2 \phi_{1 x X}-2 \phi_{1 y Y}-\Omega_{0} \psi_{1 Y}, \\
\psi_{2 x x}+\psi_{2 y y}-\left(2 x+u_{0}^{2}\right) \psi_{2}-\Omega_{0} \phi_{2 y} & =2 u_{0} \phi_{1} \psi_{1}-2 \psi_{1 x X}-2 \psi_{1 y Y}+\Omega_{0} \psi_{1 Y},
\end{aligned}
$$


with $\phi_{1}$ and $\psi_{1}$ given in Eq. (9), the terms on the right-hand sides of Eq. (12) involve terms proportional to $C^{2} e^{i 2 m_{0} y}, C_{X} e^{i m_{0} y}, C_{Y} e^{i m_{0} y}$, and $|C|^{2}$, along with the corresponding complex conjugates. We therefore write the solution to Eq. 12 as

$$
\begin{aligned}
\left(\begin{array}{c}
\phi_{2} \\
\psi_{2}
\end{array}\right)=C^{2}\left(\begin{array}{c}
A_{22}(x) \\
-i B_{22}(x)
\end{array}\right) e^{i 2 m_{0} y}+\left[C_{Y}\left(\begin{array}{c}
A_{21}(x) \\
-i B_{21}(x)
\end{array}\right)+C_{X}\left(\begin{array}{c}
i \alpha_{21}(x) \\
\beta_{21}(x)
\end{array}\right)\right] e^{i m_{0} y}+ & \\
& +|C|^{2}\left(\begin{array}{c}
A_{20} \\
B_{20}(x)
\end{array}\right)+\text { c.c. },
\end{aligned}
$$

where the equations for $A_{22}(x), B_{22}(x), \ldots$, are given by

$$
\begin{gathered}
L_{2 m_{0}}\left(\begin{array}{c}
A_{22} \\
B_{22}
\end{array}\right)=\left(\begin{array}{c}
-3 u_{0} A_{1}^{2}+u_{0} B_{1}^{2} \\
-2 u_{0} A_{1} B_{1}
\end{array}\right), \quad A_{22}, B_{22} \rightarrow 0 \text { as } x \rightarrow \pm \infty, \\
L_{m_{0}}\left(\begin{array}{c}
A_{21} \\
B_{21}
\end{array}\right)=\left(\begin{array}{c}
2 m_{0} A_{1}-\Omega_{0} B_{1} \\
2 m_{0} B_{1}-\Omega_{0} A_{1}
\end{array}\right), \quad A_{21}, B_{21} \rightarrow 0 \text { as } x \rightarrow \pm \infty, \\
L_{m_{0}}\left(\begin{array}{c}
\alpha_{21} \\
\beta_{21}
\end{array}\right)=\left(\begin{array}{c}
-2 A_{1}^{\prime} \\
-2 B_{1}^{\prime}
\end{array}\right), \quad \alpha_{21}, \beta_{21} \rightarrow 0 \text { as } x \rightarrow \pm \infty,
\end{gathered}
$$

and

$$
L_{0}\left(\begin{array}{l}
A_{20} \\
B_{20}
\end{array}\right)=\left(\begin{array}{c}
6 u_{0} A_{1}^{2}+2 u_{0} B_{1}^{2} \\
0
\end{array}\right), \quad A_{20}, B_{20} \rightarrow 0 \text { as } x \rightarrow \pm \infty .
$$

In Eqs. (13)-15), the linear operator $L_{m_{0}}$ is defined in Eq. 10a. Since there exists a non-trivial solution to the self-adjoint system (10a), for solutions to Eq. 14 to exist, the right-hand sides must each satisfy the Fredholm conditions

$$
\int_{-\infty}^{\infty}\left(A_{1}, B_{1}\right)\left(\begin{array}{c}
2 m_{0} A_{1}-\Omega_{0} B_{1} \\
2 m_{0} B_{1}-\Omega_{0} A_{1}
\end{array}\right) d x=0 \quad \text { and } \quad \int_{-\infty}^{\infty}\left(A_{1}, B_{1}\right)\left(\begin{array}{c}
-2 A_{1}^{\prime} \\
-2 B_{1}^{\prime}
\end{array}\right) d x=0 .
$$

The second condition in Eq. (16) may be seen from integrating by parts once and applying the boundary conditions in Eq. (10a). The first condition may be inferred from the fact that a solution to Eq. (14a) exists and is given by $\left(A_{21}, B_{21}\right)=\partial_{m_{0}}\left(A_{1}, B_{1}\right)$, which may be seen by differentiating Eq. 10a with respect to $m_{0}$ while noting that $d \Omega_{0} / d m_{0}=0$. This condition, along with the the normalization constraint in Eq. (11), yields the identity

$$
\int_{-\infty}^{\infty} A_{1} B_{1} d x=\frac{m_{0}}{\Omega_{0}}
$$

With Eq. 16] satisfied, we impose the additional orthogonality constraints

$$
\int_{-\infty}^{\infty}\left(A_{1}, B_{1}\right)\left(\begin{array}{l}
A_{21} \\
B_{21}
\end{array}\right) d x=0, \quad \text { and } \quad \int_{-\infty}^{\infty}\left(A_{1}, B_{1}\right)\left(\begin{array}{c}
\alpha_{21} \\
\beta_{21}
\end{array}\right) d x=0
$$

to uniquely specify $A_{21}, B_{21}, \alpha_{21}$ and $\beta_{21}$. The solutions of Eqs. 13 - 15 are depicted in Fig. 2 .

At $\mathcal{O}\left(\delta^{3}\right)$, we have that

$$
\begin{aligned}
\phi_{3 x x}+\phi_{3 y y}-\left(2 x+3 u_{0}^{2}\right) \phi_{3}+\Omega_{0} \psi_{3 y} & =R_{\phi}, \\
\psi_{3 x x}+\psi_{3 y y}-\left(2 x+u_{0}^{2}\right) \psi_{3}-\Omega_{0} \phi_{3 y} & =R_{\psi},
\end{aligned}
$$

where $R_{\phi}$ and $R_{\psi}$ contain the secular terms $S_{\phi}(x) e^{i m_{0} y}$ and $S_{\psi}(x) e^{i m_{0} y}$, respectively, along with other non-resonant terms that we need not consider. For completeness, we give the explicit expressions for the 


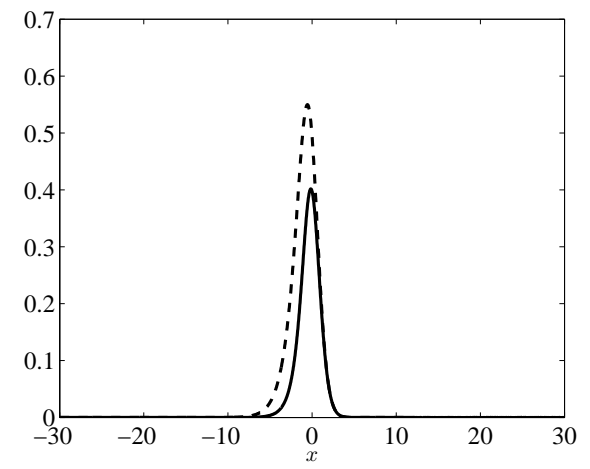

(a) $A_{1}$ and $B_{1}$

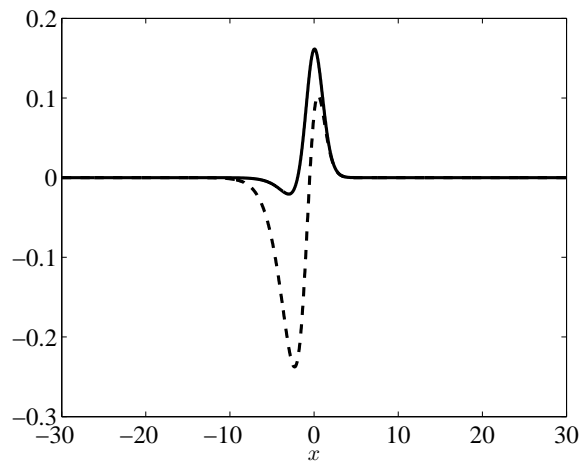

(c) $A_{21}$ and $B_{21}$

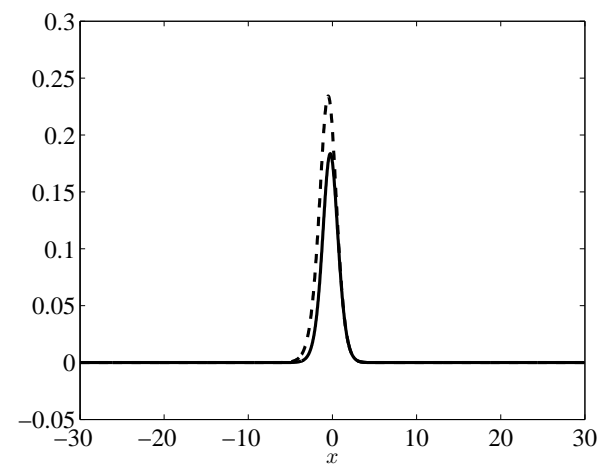

(b) $A_{22}$ and $B_{22}$

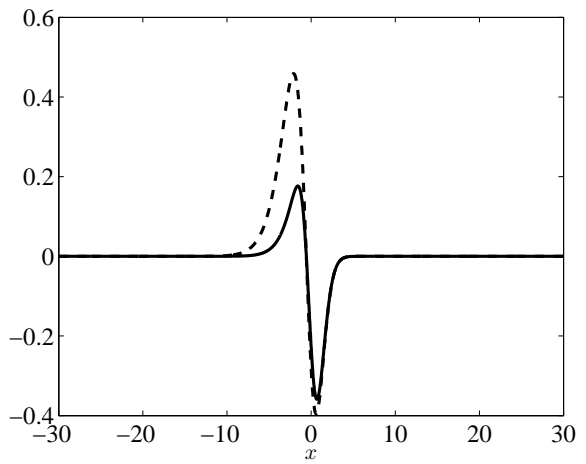

(d) $\alpha_{21}$ and $\beta_{21}$

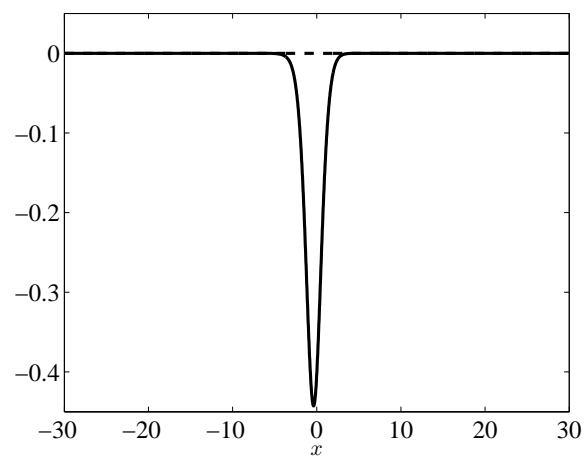

(e) $A_{20}$ and $B_{20}$

Figure 2: Solutions of Eqs. (13)-15), with $A_{1}, A_{22}, A_{21}, \alpha_{21}$ and $A_{20}$ shown in solid, and $B_{1}, B_{22}, B_{21}, \beta_{21}$ and $B_{20}$ in dashed. 
amplitudes of these secular term:

$$
\begin{gathered}
S_{\phi}=\left(\gamma i A_{1}+B_{1}\right) \frac{\partial C}{\partial T}-i\left(2 \alpha_{21}^{\prime}+A_{1}\right) \frac{\partial^{2} C}{\partial X^{2}}+\left(2 m_{0} \alpha_{21}-\Omega_{0} \beta_{21}-2 a_{21}^{\prime}\right) \frac{\partial^{2} C}{\partial X \partial Y}+ \\
+i\left(\Omega_{0} b_{21}-2 m_{0} a_{21}-A_{1}\right) \frac{\partial^{2} C}{\partial Y^{2}}-i \Omega_{2} m_{0} B_{1} C+ \\
+i\left(2 u_{0}\left(3 A_{1} a_{20}-3 A_{1} a_{22}-u_{0} B_{1} b_{22}\right)+3 A^{3}+A_{1} B_{1}^{2}\right)|C|^{2} C \\
S_{\psi}=\left(\gamma B_{1}-i A_{1}\right) \frac{\partial C}{\partial T}-\left(2 \beta_{21}^{\prime}+B_{1}\right) \frac{\partial^{2} C}{\partial X^{2}}-i\left(2 m_{0} \beta_{21}-\Omega_{0} \alpha_{21}-2 b_{21}^{\prime}\right) \frac{\partial^{2} C}{\partial X \partial Y}+ \\
+\left(\Omega_{0} a_{21}-2 m_{0} b_{21}-B_{1}\right) \frac{\partial^{2} C}{\partial Y^{2}}-\Omega_{2} m_{0} A_{1} C+ \\
+\left[2 u_{0}\left(B_{1} a_{22}-A_{1} b_{22}+B_{1} a_{20}\right)+A_{1}^{2} B_{1}+3 B_{1}^{3}\right]|C|^{2} C .
\end{gathered}
$$

The solution to Eq. (17) may then be written as $\left(\phi_{3}, \psi_{3}\right)=\left(i A_{31}(x), B_{31}(x)\right) e^{i m_{0} y}+\ldots$, where $A_{31}$ and $B_{31}$ satisfy the system

$$
L_{m_{0}}\left(\begin{array}{c}
A_{31} \\
B_{31}
\end{array}\right)=\left(\begin{array}{c}
-i S_{\phi} \\
S_{\psi}
\end{array}\right)
$$

Applying the Fredholm condition to Eq. (18)

$$
\int_{-\infty}^{\infty}\left(A_{1}, B_{1}\right)\left(\begin{array}{c}
-i S_{\phi} \\
S_{\psi}
\end{array}\right) d x=0
$$

we obtain the following amplitude equation for $C(X, Y, T)$ :

$$
\left(i \tau_{1}-\gamma \tau_{2}\right) \frac{\partial C}{\partial T}+D_{X X} \frac{\partial^{2} C}{\partial X^{2}}+i D_{X Y} \frac{\partial^{2} C}{\partial X \partial Y}+D_{Y Y} \frac{\partial^{2} C}{\partial Y^{2}}+\sigma C+\alpha|C|^{2} C=0 .
$$

The coefficients in Eq. (19) are all real, and are given by

$$
\begin{aligned}
\tau_{1}= & \frac{2 m_{0}}{\Omega_{0}}>0, \quad \tau_{2}=1, \quad \sigma=\frac{2 m_{0}^{2}}{\Omega_{0}} \Omega_{2}>0 \\
\alpha= & -\int_{-\infty}^{\infty} A_{1}\left[2 u_{0}\left(-3 A_{1} A_{22}+3 A_{1} A_{20}-B_{1} B_{22}\right)+3 A_{1}^{2}+A_{1} B_{1}^{2}\right]+ \\
& B_{1}\left[2 u_{0}\left(-A_{1} B_{22}+A_{20} B_{1}+A_{22} B_{1}\right)+A_{1}^{2} B_{1}+3 B_{1}^{3}\right] d x>0 \\
D_{X X}= & \int_{-\infty}^{\infty} A_{1}\left[-2 \alpha_{21}^{\prime}-A_{1}\right]+B_{1}\left[-2 \beta_{21}^{\prime}-B_{1}\right] d x \approx 0 \\
D_{X Y}= & \int_{-\infty}^{\infty} A_{1}\left[2 A_{21}^{\prime}-2 m_{0} \alpha_{21}+\Omega_{0} \beta_{21}\right]+B_{1}\left[2 B_{21}^{\prime}-2 m_{0} \beta_{21}+\Omega_{0} \alpha_{21}\right] d x \approx 0 \\
D_{Y Y}= & -\int_{-\infty}^{\infty} A_{1}\left[-A_{1}-2 m_{0} A_{21}+\Omega_{0} B_{21}\right]+B_{1}\left[-B_{1}-2 m_{0} B_{21}+\Omega_{0} A_{21}\right] d x>0 .
\end{aligned}
$$

We note that $\sigma>0$ since we assume that the system is above threshold; i.e., $\Omega_{2}>0$ so that $\Omega>\Omega$. With the normalization (11) and values of $m_{0}$ and $\Omega_{0}$ given in Eq. 10b, we numerically obtain the following values for the coefficients, accurate to the fifth decimal place:

$$
\begin{gathered}
\tau_{1} \approx 0.87884, \quad \tau_{2}=1, \quad \sigma \approx 0.97671 \Omega_{2}, \quad \alpha \approx 0.62184 \\
D_{Y Y} \approx 0.67615, \quad D_{X Y} \sim 10^{-12}, \quad \text { and } \quad D_{X X} \sim 10^{-6}
\end{gathered}
$$

The values above show that, remarkably, $D_{X X}$ and $D_{X Y}$ are very close to zero. While they may or may not be exactly zero, for all practical purposes hereafter, we will indeed set them to 0 . In this way, the 
two-dimensional dynamics in the weakly nonlinear regime of Eq. (3) reduce to dynamics along only one dimension. The same reduction was observed for the one-dimensional dynamics of edge modes in a twodimensional NLSE in the presence of a honeycomb potential [2]. Indeed, this may be an indication (exactly, or just approximately so) of an effective "topological protection" [3], a theme of intense recent interest in the physics community [35. The reason we indicate the potentially approximate nature of the topological protection for our (toroidal) domain strip is that eventually the ensuing vortices escape inwards towards the center of the domain. Nevertheless, exploring this aspect in the context of the present work further is an especially appealing aspect for further study.

Further proceeding with our reduction, by neglecting the $D_{X X}$ and $D_{X Y}$ terms as indicated above, our analysis yields the one-dimensional amplitude equation for the envelope $C(Y, T)$

$$
\left(i \tau_{1}-\gamma\right) \frac{\partial C}{\partial T}+D_{Y Y} \frac{\partial^{2} C}{\partial Y^{2}}+\sigma C+\alpha|C|^{2} C=0 .
$$

We make one remark regarding the scaling of $\gamma$ with respect to $\delta$. Due to the $C \rightarrow-C$ invariance of Eq. (9), the largest term omitted from the amplitude equation (21) is the quintic term $|C|{ }^{4} C$. This term is of $\mathcal{O}\left(\delta^{2}\right)$ with respect to the rest of the terms in Eq. (21). Therefore, while we assumed in the analysis that $\gamma=\mathcal{O}(1)$ with respect to $\delta$, we in fact only require that $\gamma \gg \delta^{2}$ for Eq. (21) to be valid. Further, as we show in the next section, when $\gamma$ is small, it is responsible only for the growth of low wavenumber perturbations of a spatially uniform state, and the dissipation of high wavenumber perturbations. The symmetry breaking mechanism in Eq. (21) that initiates vortex formation in the full system (3) occurs on an $\mathcal{O}(1)$ time scale independent of $\gamma$. As such, Eq. 21) retains all the orders required to accurately describe the weakly nonlinear dynamics of the full system.

To examine the validity of the weakly nonlinear theory, we solved the two-dimensional system (3) numerically on the domain $x \in[-7.5,22.5], y \in\left[-80 \pi / m_{0}, 80 \pi / m_{0}\right]$ so that exactly 80 wavelengths of the critical mode $m_{0}$ fit inside the domain of length $L_{y}=160 \pi / m_{0}$. The initial conditions were taken to be of the form given in Eq. (9) with an envelope randomly perturbed from unity. That is $C(Y, 0)=1+0.01 * \operatorname{rand}(y)$, where $\operatorname{rand}(y)$ takes on a uniformly distributed random value between 0 and 1 at each discrete point in $y$. The parameters $\gamma$ and $\delta$ were taken to be $\gamma=0.01$ and $\delta=0.04$. While realistic values of $\gamma$ are typically smaller than 0.01 , this was purely for demonstration purposes and similar results are obtained for smaller, more realistic, values of $\gamma$. We also simultaneously solved the one-dimensional amplitude equation (21) on the domain $Y \in\left[-80 \delta \pi / m_{0}, 80 \delta \pi / m_{0}\right]$; that is, on a domain of length $L=\delta L_{y}$, consistent with the scaling in Eq. (7b). The comparison of the two sets of results is shown in Fig. 3. Each panel 3(a) 3(f) is arranged into a left, center, and right column. In the center column of each figure, we show a surface plot of $|w|$, while in the right column, we show a surface plot of $\Im(w)=\psi$. Blue (red) regions indicate small (large) values in the plotted quantity. In the two plots that make up the leftmost column, we show in red a slice of $|\phi| / \delta$ (top) and $|\psi| / \delta$ (bottom) taken near $x=0$, corresponding to the vicinity of the Thomas-Fermi radius where vortices first form in the original system (2). Here, $\phi$ and $\psi$ are the real and imaginary parts of the perturbation, respectively, and obey Eq. (5). In black, we plot the envelope obtained by solving Eq. (21). We observe excellent agreement, indicating that the two-dimensional dynamics of Eq. (3) in the weakly nonlinear regime can indeed be captured by the one-dimensional amplitude Eq. 21.

The initial symmetry breaking is shown in Fig. 3(b) where the uniform $C=1$ state (see Fig. 3(a)p evolves into a spatially periodic state. This is due to a MI in Eq. (21), which will be discussed in Sec. 4 . Over a relatively shorter time scale, the envelope enters the weakly nonlinear regime, oscillating between a slightly localized state (see Fig. 3(c) and a highly localized state (see Fig. 3(d)). This stage can still be accurately captured by our effective one-dimensional model. It then enters the fully nonlinear regime (see Fig. 3(e)p as two of the localized regions become dominant and results in the formation of two vortices that then get pulled into the bulk of the condensate (see Fig. 3(f) . As seen in Figs. 3(e) and 3(f), these vortices manifest as dips in the surface of $|w|$. In this regime, the weakly nonlinear results are no longer applicable. However, it is clear that the initial MI (see Fig. 3(b) in the one-dimensional amplitude Eq. 21) is the symmetry breaking mechanism responsible for the initiation of the process leading to the formation of vortices in the two-dimensional system (3).

To show that Eq. 21) is equivalent to (a dissipative variant of) the self-focusing NLSE when $\gamma=0$, we 

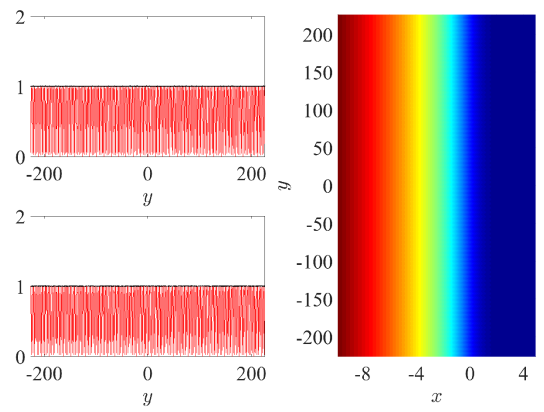

(a) $t=0$
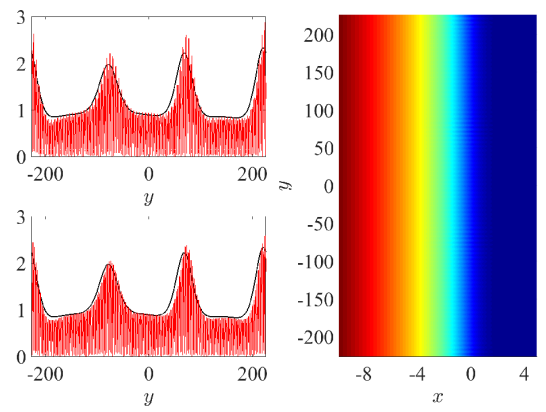

(c) $t=6750$
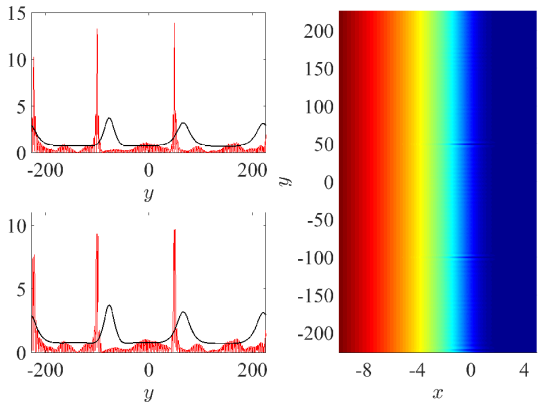

(e) $t=8859.375$
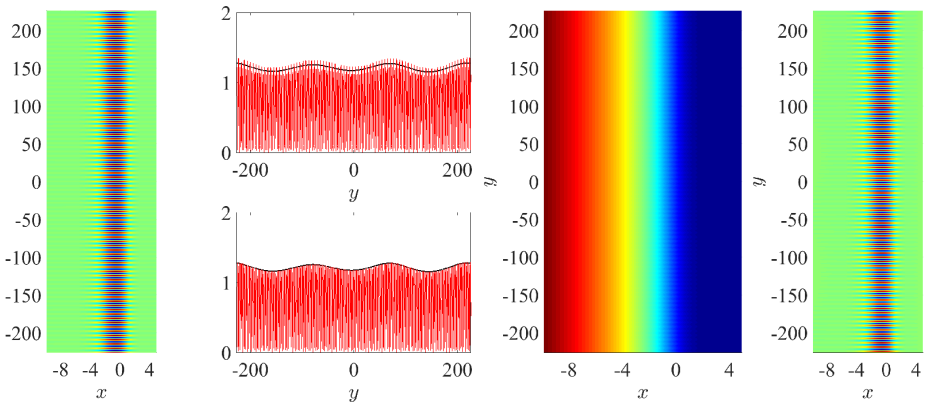

(b) $t=5343.75$
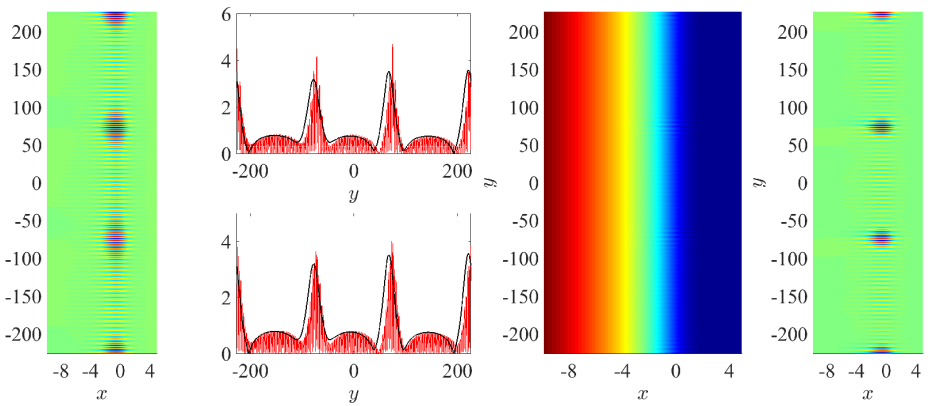

(d) $t=7171.875$
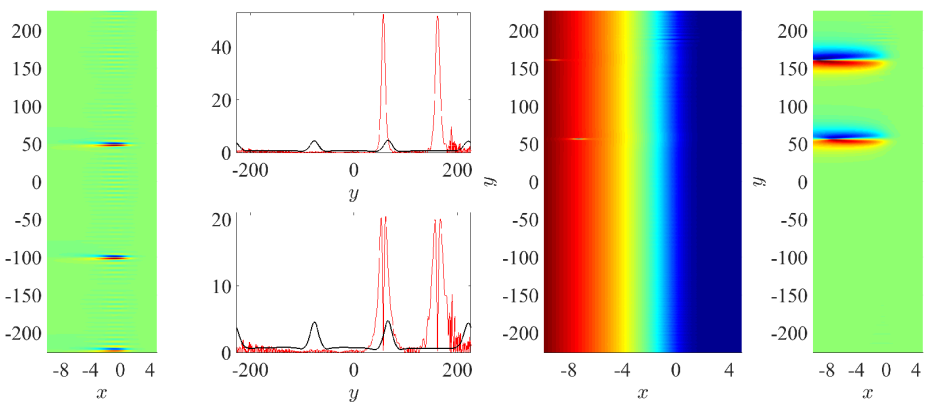

(f) $t=9421.875$

Figure 3: Evolution of the perturbation at the periphery of the atomic cloud. Each panel (a)-(f) is arranged into a left, center, and right column. The center and right columns depict, respectively, the amplitude and imaginary part of the solution, where blue (red) regions indicate small (large) values. The left columns depict the comparison of the one-dimensional dynamics of the amplitude Eq. 21) (black) versus the dynamics of the full two-dimensional system Eq. (3) (red). The red is taken from an $x$-slice of $\phi$ (top subpanel) and $\psi$ (bottom subpanel), the real and imaginary parts of the perturbation, respectively. Starting from random perturbations of the envelope about unity (a), the third mode is selected (b). The pattern oscillates between a slightly more localized state (c) and a highly localized state (d), before entering the fully nonlinear regime (e) where two vortices are nucleated and pulled into the bulk (f). 
introduce the rescaled variables

$$
T=\tau_{1}\left(1+\widetilde{\gamma}^{2}\right) \tau, \quad Y=\sqrt{D_{Y Y}} \eta, \quad C(Y, T)=\sqrt{\frac{2}{\alpha}} B(\eta, \tau) ; \quad \text { where } \quad \widetilde{\gamma} \equiv \frac{\gamma}{\tau_{1}},
$$

to obtain

$$
\frac{\widetilde{\gamma}-i}{1+\widetilde{\gamma}^{2}} B_{\tau}=B_{\eta \eta}+\sigma B+2|B|^{2} B
$$

Lastly, we multiply Eq. (23) across by $\widetilde{\gamma}+i$ and scale out the rotation by letting

$$
B(\eta, \tau)=e^{i \sigma \tau} A(\eta, \tau)
$$

to obtain

$$
A_{\tau}=(\widetilde{\gamma}+i) A_{\eta \eta}+\sigma \widetilde{\gamma} A+2(\widetilde{\gamma}+i)|A|^{2} A .
$$

Setting $\widetilde{\gamma}=0$ in Eq. 25), we see that Eq. (21) is equivalent to the self-focusing nonlinear NLSE. Due to rotation $\left(A \rightarrow A e^{i \theta}\right)$ and dilation $\left(A \rightarrow \lambda A, \eta \rightarrow \lambda \eta\right.$, and $\left.\tau \rightarrow \lambda^{2} \tau\right)$ invariance the self-focusing NLSE admits a family of one-soliton solutions of the form

$$
A_{s}(\eta, \tau ; v, r)=r \operatorname{sech}[r(\eta+2 v \tau)] e^{-i \theta(\eta, \tau)} ; \quad \theta(\eta, \tau)=v \eta+\left(v^{2}-r^{2}\right) \tau .
$$

For $\widetilde{\gamma} \ll 1$ in Eq. 225 , a perturbation analysis invoking additional translation $\left(\eta \rightarrow \eta_{0}\right)$ and Galilean $\left(A \rightarrow A e^{i c \eta-i c^{2} \tau}\right.$ and $\left.\eta \rightarrow \eta-2 c \tau\right)$ symmetries leads to a coupled system of equations for the slow time evolution of $r(\widetilde{\gamma} \tau)$ and $v(\widetilde{\gamma} \tau)$ (see, e.g., Refs. [12, 13, 11, 20, for details).

Based on the time scales in Figs. 3, we find that, once vortices form, they quickly enter the fully nonlinear regime. As such, a detailed analysis of the evolution of a localized soliton solution in the amplitude equation, the latter of which is only valid in the weakly nonlinear regime of Eq. (3), is not particularly useful. We are presently not aware of a technique (aside from the detailed numerical simulations, such as those of Fig. 3(e)$3(\mathrm{f})$ that could capture this second stage of (large amplitude) symmetry breaking. Instead, we focus on the initial symmetry breaking mechanism in Eq. (21) that initiates the formation of vortices in Eq. (3). As seen in Fig. 3(b), this symmetry breaking does occur in the weakly nonlinear regime of Eq. (3), and is the result of a MI in Eq. (21). We analyze this instability in the following section.

\section{Modulational instability}

In this section we analyze the MI of a spatially homogeneous time-dependent solution of Eq. 21. The analysis follows that of Ref. [33; see also Ref. [36. To obtain an exact solution of Eq. 25] without the spatial term, we take the ansatz

$$
A=A_{0}(\tau)=f(\tau) e^{i g(\tau)},
$$

where the functions $f$ and $g$ satisfy the ODE's

$$
\begin{gathered}
f^{\prime}=\widetilde{\gamma}\left[\sigma f+2 f^{3}\right], \quad f(0)=|A(0)| ; \\
g^{\prime}=2 f^{2}, \quad g(0)=\arg (A(0)) .
\end{gathered}
$$

The system (26) is solved analytically, yielding

$$
f(\tau)=\frac{\sqrt{\sigma}}{\sqrt{-2+c_{1} e^{-2 \sigma \widetilde{\gamma} \tau}}} ; \quad g(\tau)=-\frac{1}{2 \widetilde{\gamma}} \log \left[-2+c_{1} e^{-2 \sigma \widetilde{\gamma} \tau}\right]-\sigma \tau+c_{2},
$$

where

$$
c_{1} \equiv 2+\frac{\sigma}{|A(0)|^{2}} ; \quad c_{2} \equiv \frac{1}{2 \gamma} \log \left(\frac{\sigma}{|A(0)|^{2}}\right)+\arg (A(0)) .
$$

A spatially homogeneous solution $C_{0}(T)$ of Eq. 21) is then given by Eq. (27) and the scalings in Eqs. (22) and (24). In particular, we calculate that

$$
\left|C_{0}\right|^{2}=\frac{2 \sigma}{\alpha} \frac{1}{-2+c_{0} e^{-\frac{2 \tilde{\gamma}}{1+\tilde{\gamma}^{2}} \frac{\sigma}{\tau_{1}} T}} ; \quad c_{0} \equiv 2+\frac{2 \sigma}{\alpha\left|C_{0}(0)\right|^{2}} .
$$


In what follows, we let $C \rightarrow u, T \rightarrow t, Y \rightarrow y$, and $D_{Y Y} \rightarrow D$ for cleaner notation.

To analyze the stability of $u_{0}(t)$, we introduce the perturbation

$$
u(y, t)=u_{0}(t)(1+\varepsilon w(t) \cos q y) ; \quad \varepsilon \ll 1 .
$$

Substituting Eq. (29) into Eq. (21) and equating coefficients of $\cos q y$ at the leading order in $\varepsilon$, we obtain

$$
\left(i \tau_{1}-\gamma\right)\left[\frac{u_{0}^{\prime}}{u_{0}} w+w^{\prime}\right]-q^{2} D w+\sigma w+\alpha\left|u_{0}\right|^{2}[\bar{w}+2 w]=0 .
$$

Next, noting that $u_{0}(t)$ is a solution of Eq. (21), yields

$$
\left(i \tau_{1}-\gamma\right) \frac{u_{0}^{\prime}}{u_{0}}=-\sigma-\alpha\left|u_{0}\right|^{2} .
$$

Substituting Eq. (31) into Eq. (30) and simplifying yields

$$
\left(i \tau_{1}-\gamma\right) w^{\prime}-\left[q^{2} D-\left|u_{0}\right|^{2}\right] w-\alpha\left|u_{0}\right|^{2} \bar{w}=0 .
$$

Now, letting $w=w_{r}+i w_{i}$ and separating real and imaginary parts, yields the matrix eigenvalue problem

$$
\frac{d}{d t}\left(\begin{array}{c}
w_{r} \\
w_{i}
\end{array}\right)=\frac{1}{\gamma^{2}+\tau_{1}^{2}}\left(\begin{array}{cr}
\gamma\left[(1+\alpha)\left|u_{0}\right|^{2}-q^{2} D\right] & -\tau_{1}\left[(1-\alpha)\left|u_{0}\right|^{2}-q^{2} D\right] \\
\tau_{1}\left[(1+\alpha)\left|u_{0}\right|^{2}-q^{2} D\right] & \gamma\left[(1-\alpha)\left|u_{0}\right|^{2}-q^{2} D\right]
\end{array}\right)\left(\begin{array}{l}
w_{r} \\
w_{i}
\end{array}\right) .
$$

The system (32) is non-autonomous due to the time-dependence of $\left|u_{0}\right|^{2}$ given in Eq. (28) (recall $C_{0} \rightarrow u_{0}$ ). However, since $\gamma \ll 1$, we observe by Eq. $(26)$ that $\left|u_{0}(t)\right|$ evolves on an asymptotically slow time scale as long as $\left|u_{0}\right| \ll \gamma^{-1 / 3}$. The ODE system (32) therefore takes the form $\mathbf{w}^{\prime}=M(\gamma t) \mathbf{w}$, where $M(\gamma t)$ is the two-by-two matrix in Eq. 32 with entries that evolve slowly on an $\mathcal{O}(\gamma)$ time scale. This suggests a WKB ansatz for $\mathbf{w}$ of the form

$$
\mathbf{w}=\mathbf{v}(s) e^{r(s) / \gamma} ; \quad s=\gamma t .
$$

Substituting Eq. (33) into Eq. (32) and collecting terms at leading order in $\gamma$, we find that $d r / d s$ satisfies the stationary eigenvalue problem $M \mathbf{v}=(d r / d s) \mathbf{v}$. We may thus identify $d r / d s$ with the eigenvalues of $M$ computed with its entries frozen in time. Therefore, $\mathbf{w}=\left(w_{r}, w_{i}\right)^{T}$ grows (decays) when the eigenvalue of $M$ with the largest real part lies on the right (left) half-plane. Scenarios in which the aforementioned eigenvalue slowly crosses from the left half-plane into the right half-plane may often lead to the phenomenon of delayed bifurcations [6, 23. That is, the bifurcation may not become observable until the slowly varying control parameter responsible for the eigenvalue crossing is an $\mathcal{O}(1)$ distance past the linear stability threshold. This phenomenon is absent here since, as we will observe below, the eigenvalue crossing is not slow. As such, we will say that an instability in Eq. (32) has been triggered when the largest eigenvalue acquires zero real part.

The eigenvalue of the matrix in Eq. (32) with largest real part is given by

$$
\lambda(q)=\frac{\operatorname{tr}}{2}+\sqrt{\frac{\operatorname{tr}^{2}}{4}-\operatorname{det}}
$$

where

$$
\operatorname{tr} \equiv \frac{2 \gamma\left[\left|u_{0}\right|^{2}-q^{2} D\right]}{\gamma^{2}+\tau_{1}^{2}}, \quad \text { and } \quad \operatorname{det} \equiv \frac{\left[(1+\alpha)\left|u_{0}\right|^{2}-q^{2} D\right]\left[(1-\alpha)\left|u_{0}\right|^{2}-q^{2} D\right]}{\gamma^{2}+\tau_{1}^{2}} .
$$

In the limit of small $\gamma$, we see that $\lambda(q)$ is $\mathcal{O}\left(\left|u_{0}\right|^{2}\right)$ and positive when $q^{2}$ lies in the interval $\left(q_{-}^{2}, q_{+}^{2}\right)$, with $q_{ \pm}^{2} \equiv(1 \pm \alpha)\left|u_{0}\right|^{2} / D$. This band of positively growing wavenumbers is what is responsible for the symmetry breaking mechanism that initiates the formation of vortices. To the left of this band, $\Re(\lambda(q))$ is $\mathcal{O}(\gamma)$ and positive, while to the right of this band, $\Re(\lambda(q))$ is $\mathcal{O}\left(\gamma q^{2}\right)$ and negative. We thus see that the presence of small $\gamma>0$ is responsible for amplification of the low wavenumbers and dissipation of the high wavenumbers. The band of instability, and its $\mathcal{O}\left(\left|u_{0}\right|^{2}\right)$ positive growth rate, would be present even in the case of $\gamma=0$. However, small $\gamma$ still influences pattern formation in Eq. (21) through the growth of $\left|u_{0}\right|^{2}$ by shifting the band of instability towards larger wavenumbers. On a finite domain of length $L$, in which the shortest 


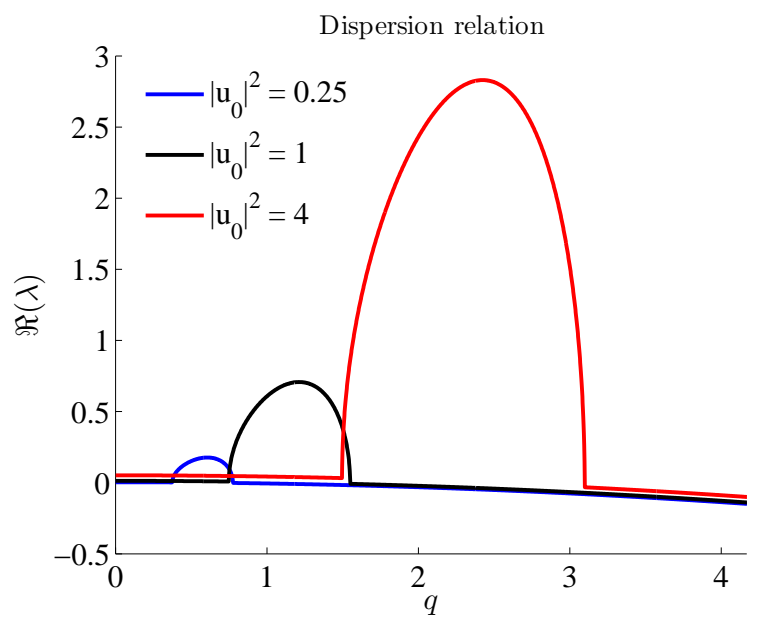

(a) $\Re(\lambda)$ vs $q$

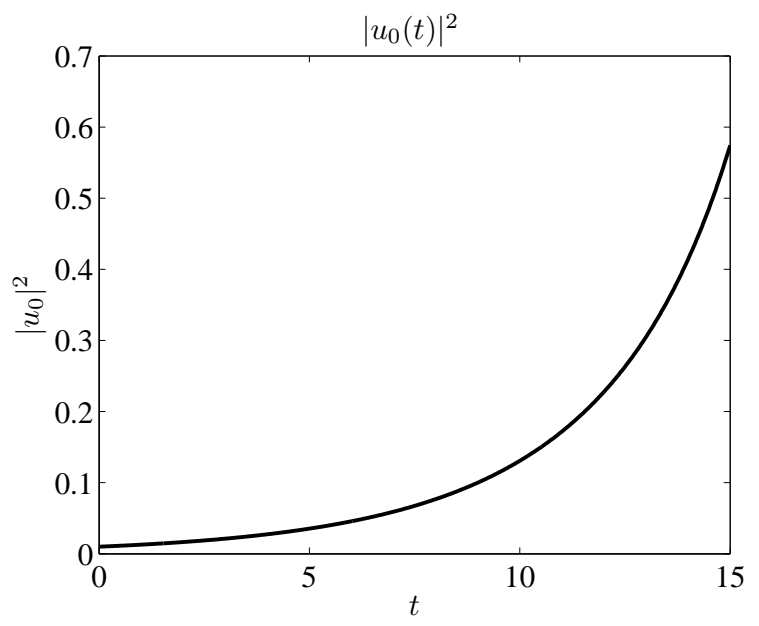

(b) $\left|u_{0}\right|^{2}$ vs $t$

Figure 4: (a) The dispersion relation given by Eq. (34) for various values of $\left|u_{0}\right|^{2}(0.25,1$, and 4 from left to right) as indicated in the legend. As $\left|u_{0}\right|^{2}$ increases, the band of unstable wavenumbers broadens, shifts to the right, and acquires larger growth rates. The height and shape of the bands depend only weakly on $\gamma$ when $\gamma$ is small. (b) The time evolution of $\left|u_{0}\right|^{2}$ given by Eq. (28). The parameters are $\gamma=0.01$ and $\Omega_{2}=1$.

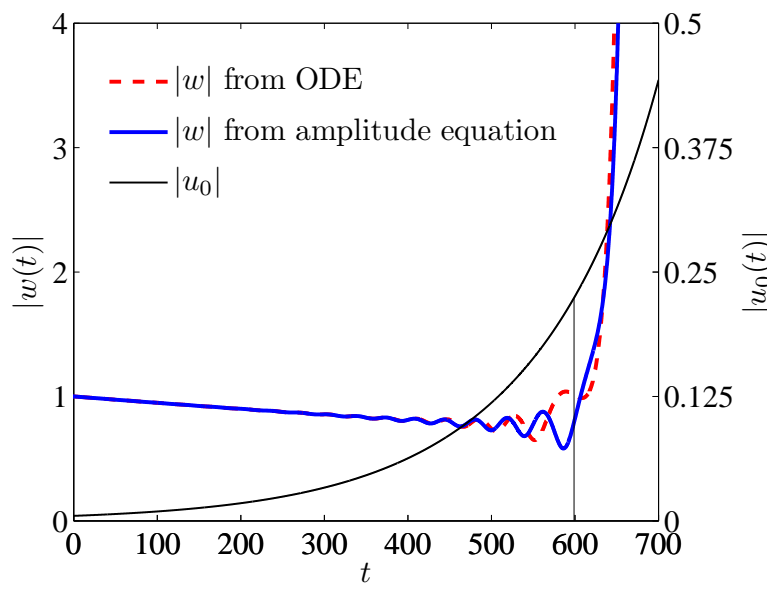

(a) amplitude equation vs. ODE

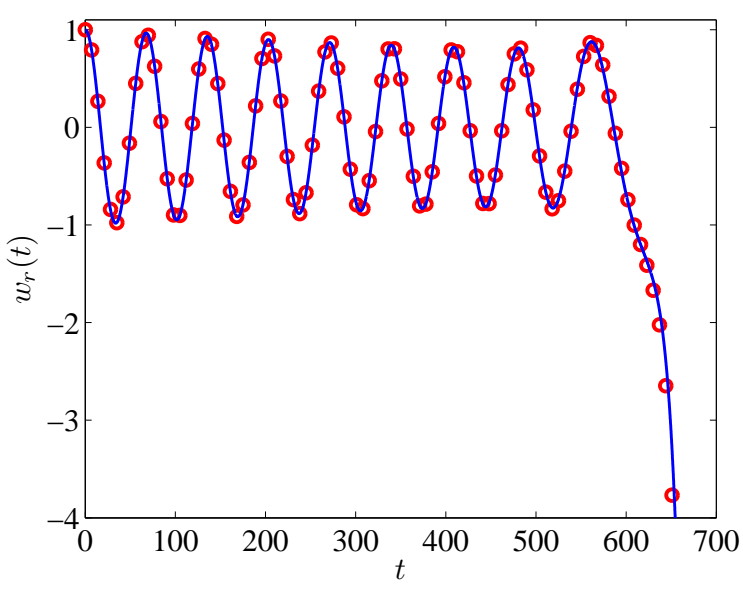

(b) full PDE system vs. amplitude equation

Figure 5: (a) Growth rate of the perturbation at the periphery of the atomic cloud. The thick solid blue line (using the left axis) depicts $|w(t)|$ as numerically extracted from the PDE solution of Eq. (21) using the perturbation prescribed by Eq. (29). The dashed red line depicts $|w(t)|$ computed from the linearized ODE (32). The light solid line black depicts $\left|u_{0}(t)\right|$ (using the right axis). Both the ODE and PDE dynamics show that $|w(t)|$ decays initially until $\left|u_{0}(t)\right|$ has increased enough so that the smallest admissible wavenumber acquires positive growth rate. This happens approximately when $\left|u_{0}(t)\right|$ reaches 0.224 (vertical line). While the ODE dynamics seems to exhibit a cumulative phase error with respect to the PDE dynamics, it does accurately predict when the instability is triggered. (b) Comparison of $\Re(w)$ as extracted from the time evolution of the full two-dimensional system (3) (red circles) and that extracted from the same time evolution of the amplitude equation (21) (blue solid) as in (a). The horizontal axis in both figures is of slow time. The parameters are $\delta=0.04, \gamma=0.005, \delta L_{y} \approx 18.09$, and $\Omega_{2}=1$. 
admissible wavelength $q_{1}=2 \pi / L$ may initially lie to the right of the band of instability, positive $\gamma$ will cause the band to drift rightwards and eventually trigger the instability when $q_{+}^{2}=q_{1}^{2}$. This occurs when $\left|u_{0}\right|=\mathcal{O}(1)$. To see why this precludes the delayed bifurcations, we observe that as soon as $\lambda_{1}$ acquires positive growth rate, its growth rate is $\mathcal{O}(1)$ positive. Therefore there is no slow crossing of the eigenvalue, and hence no delay.

It is important to note that the larger the $L$, where $L=\delta L_{y}$, the greater the number of wavelengths of the unstable mode(s) the domain can contain. Relating this back to the original system (3), the farther the rotation frequency $\Omega$ is set above threshold, the more localized regions form in the weakly nonlinear regime. This may lead to more vortices in the fully nonlinear regime being pulled into the bulk. The dependence of $\lambda(q)$ on $\left|u_{0}\right|^{2}$, along with the time evolution of $\left|u_{0}\right|^{2}$, are shown in Figs. 4(a) and 4(b) respectively.

We illustrate the theory using Fig. 3, where the spatially homogeneous state being perturbed is $\left|u_{0}\right|=1$. The domain length is $\delta L_{y} \approx 18.09$ so that, by Fig. $4(\mathrm{a})$, the third $(q \approx 1.04)$ and the fourth $(q \approx 1.39)$ modes are the two modes that lie in the band of instability, both having similar growth rates. Consistent with the theory, three bumps appear in Fig. 3(b). Four bumps may also form given the same parameter set and different random initial conditions.

Lastly, we demonstrate how the growth of $\left|u_{0}\right|$ due to positive $\gamma$ can intrinsically trigger a MI. On a domain of length $L \approx 18.09$, we solve Eq. (21) with $\left|u_{0}(0)\right|=0.005$ and $\gamma=0.005$. The smallest admissible wavenumber in this domain is $q_{1}=2 \pi / L \approx 0.3473$. According to Eq. (34), this wavenumber initially lies to the right of the band of instability. Therefore, the spatially homogeneous state is initially stable. However, as $\left|u_{0}\right|$ increases according to Eq. (28), the band of instability drifts to the right. When $\left|u_{0}\right|$ increases to approximately $\left|u_{0}\right| \approx 0.224, \Re\left(\lambda\left(q_{1}\right)\right)$ becomes positive, and the mode $\cos \left(q_{1} y\right)$ begins to grow. This is illustrated in Fig. 5(a), where we initialize $u$ as $u(y, 0)=\left|u_{0}(0)\right|\left(1+2 \times 10^{-5} \cos \left(q_{1} y\right)\right)$. The figure depicts with a thick solid blue line the evolution of $|w(t)|$ as numerically extracted from the PDE solution of Eq. (21) using the perturbation prescribed by Eq. (29). The dashed red line depicts the evolution of $|w(t)|$ as computed from the linearized ODE (32). The initial (oscillatory) decay is due to $q_{1}$ lying initially to the right of the instability band so that $\lambda\left(q_{1}\right)$ lies in the left half-plane with $\Im\left(\lambda\left(q_{1}\right)\right) \neq 0$. When $\left|u_{0}(t)\right|$ (light solid black line and right axis) increases to approximately 0.224 (thin vertical line), $\Re\left(\lambda\left(q_{1}\right)\right.$ ) becomes positive real so that the amplitude of the perturbation begins to increase monotonically, verifying the theory. While the ODE prediction appears to exhibit a cumulative error in the phase with respect to the PDE dynamics, it does accurately predict when the dynamically instability is triggered.

We next verify that this intrinsic triggering predicted by the MI analysis is also present in the full twodimensional system (3). In Fig. 5(b)] we compare $\Re(w)$ as extracted from the time evolution of the full PDE system (3) (red circles) and that extracted from the same time evolution of the amplitude equation (21) (blue solid) as in Fig. 5(a). The parameters in the full PDE were taken to be $\delta=0.04, \gamma=0.005, \delta L_{y} \approx 18.09$, and $\Omega_{2}=1$. We observe excellent agreement between the two dynamics. As in Fig. 5(a). Fig. 5(b) exhibits a slow oscillatory decay followed by fast monotonic growth starting near $t=600$. Note that the independent variable in the horizontal axis in both figures is the rescaled slow time $T=\delta^{2} t$, which has been relabeled $t$ in accordance with the notation change $T \rightarrow t$ in this section.

To extract $\Re(w)$ from the full PDE data, we first calculate from Eq. (9) that for a given slice $x=x_{0}$,

$$
\frac{\psi_{1}\left(x_{0}, y\right)}{2 B\left(x_{0}\right)}=C_{r}(Y, T) \cos \left(m_{0} y\right)-C_{i}(Y, T) \sin \left(m_{0} y\right),
$$

where we have defined $C_{r} \equiv \Re(C), C_{i} \equiv \Im(C)$, and $Y=\delta y$ and $T=\delta^{2} t$ are the slow space and time variables, respectively. Because of the separation of scales between $y$ and $Y$ in Eq. (35), the quantity $\psi_{1}\left(x_{0}, y\right) /\left(2 B\left(x_{0}\right)\right)$ to leading order takes the form of a slowly modulated phase-shifted cosine of frequency $m_{0}$, the envelope of which is given by $|C(Y, T)|$. Next, we calculate from Eq. (29) with $u \rightarrow C, t \rightarrow T$, and $y \rightarrow Y$, that

$$
\frac{1}{2 \varepsilon} \frac{|C(Y, T)|^{2}}{\left|C_{0}(T)\right|^{2}}=\frac{1}{2 \varepsilon}+\Re(w) \cos q Y+\mathcal{O}(\varepsilon) .
$$

Here, the evolution of $\left|C_{0}(T)\right|^{2}$ is given analytically by Eq. $(28)$. Thus, to compute $\Re(w)$, we need only calculate numerically the envelope of the quantity $\psi_{1}\left(x_{0}, y\right) /\left(2 B\left(x_{0}\right)\right.$, which yields $|C|$. The value of $\Re(w)$ may then be extracted by numerically computing the amplitude of the left-hand side of Eq. (36), yielding the curve marked by red circles in Fig. 5(b). This example shows that the phenomenon, predicted by the 
MI analysis, of initial decay of a spatial perturbation followed by growth persists not only in the reduced amplitude equation, but also in the original two-dimensional PDE system.

\section{Discussion}

In the present work, we have revisited the long studied (not only theoretically and numerically, but importantly also experimentally) problem of the formation of vortices in the presence of rotation. We have argued that while a vast literature exists on the subject, there are still various gaps in our understandings of this process, including among other things the weakly (and strongly) nonlinear emergence of a single (or a few) vortices that eventually travel inward, settling towards the center of the domain. In order to shed light in the weakly nonlinear aspect within this process, we have derived a one-dimensional effective amplitude equation as a reduction of a dissipative variant of the self-defocusing two-dimensional GPE with a harmonic trap under rotation. Remarkably, this equation turns out to be a self-focusing dissipative variant of the GPE. The latter has been shown to undergo modulational instabilities and symmetry breakings that eventually result in the formation of solitons that lead to the appearance of the vortices drawn inwards in the original (full) problem. This is due to two separate symmetry breaking processes. The first, attributed to a linear (modulational) instability of a vortex-free, homogeneous steady state of the dissipative GPE as the rotation is increased above a threshold, leads to a large number of "small vortices" nucleating near the edge of the condensate cloud. The second, which we can monitor numerically, but which is beyond the realm of our weakly nonlinear theory, selects a fraction of these small vortices and pulls them into the bulk of the condensate. Not only were we able to derive an effectively one-dimensional equation describing the weakly nonlinear state (its one-dimensionality hinting at an approximate topological insulation of the system's boundary), but we were also able to quantify the modulational instability and illustrate that its temporal and spatial scales coincide with the emergence of the pattern formation within the full PDE system. While we could not capture the final highly nonlinear step of this destabilization and symmetry breaking process analytically, our numerical computations shed considerable light to it. Nevertheless, the latter would be an extremely intriguing problem for future study. While the specific pattern selection might be the most difficult step to tackle, it would also be interesting to perform an analysis along the lines of Ref. [40] to derive a system of equations of motion for the vortices as they move into the bulk.

Another key problem worth exploring, as indicated in the introduction, is the reconciliation of the surface dynamical picture put forth by Ref. [5] (see also, e.g., for a recent exposition, Ref. [10] and our earlier work of Ref. [8]) and the bulk hydrodynamic approach of Ref. [34. Lastly, it would also be relevant to perform an analysis of vortex formation in the GPE with an anisotropic potential [24]. In the isotropic case considered here, the initial instability leads to a uniform formation of small vortices all around the edge of the condensate cloud. In contrast, in the anisotropic case, this uniformity is expected to be broken. An analysis could be performed to determine where the first vortices are nucleated, and what the subsequent vortex selection mechanism is. These problems are currently under study and relevant progress will be reported in future publications.

\section{Acknowledgments}

J.C.T. was supported by an AARMS Postdoctoral Fellowship. P.G. Kevrekidis was supported by NSFDMS-1312856, as well as from the US-AFOSR under grant FA950-12-1-0332, and the ERC under FP7, Marie Curie Actions, People, International Research Staff Exchange Scheme (IRSES-605096). P.G.K.'s work at Los Alamos is supported in part by the U.S. Department of Energy. T.K. was supported by NSERC Discovery Grant No. RGPIN-33798 and Accelerator Supplement Grant No. RGPAS/461907. R.C.G. gratefully acknowledges the support of NSF-DMS-1309035.

\section{References}

[1] M. Ablowitz and P. Clarkson, Solitons, nonlinear evolution equations and inverse scattering, vol. 149, Cambridge university press, 1991. 
[2] M. Ablowitz, C. Curtis, And Y.-P. MA, Adiabatic dynamics of edge waves in photonic graphene, arXiv:1411.6598, (2014).

[3] M. Ablowitz And Y.-P. MA, Strong transmission and reflection of edge modes in bounded photonic graphene, arXiv:1506.04708, (2015).

[4] J. Abo-Shaeer, C. Raman, J. Vogels, and W. Ketterle, Observation of vortex lattices in boseeinstein condensates, Science, 292 (2001), pp. 476-479.

[5] J. Anglin, Local vortex generation and the surface mode spectrum of large bose-einstein condensates, Phys. Rev. Lett., 87 (2001), p. 240401.

[6] S. BAer, T. ERneux, And J. Rinzel, The slow passage through a hopf bifurcation: delay, memory effects, and resonance, SIAM Journal on Applied mathematics, 49 (1989), pp. 55-71.

[7] P. Blakie, A. Bradley, M. Davis, R. Ballagh, and C. Gardiner, Dynamics and statistical mechanics of ultra-cold Bose gases using c-field techniques, Advances in Physics, 57 (2008), pp. 363455 .

[8] R. Carretero-González, P. Kevrekidis, and T. Kolokolnikov, Vortex nucleation in a dissipative variant of the nonlinear Schrödinger equation under rotation, arXiv:1412.0615, (2014).

[9] F. Dalfovo, S. Giorgini, M. Guilleumas, L. Pitaevskit, and S. Stringari, Collective and single-particle excitations of a trapped bose gas, Phys. Rev. A, 56 (1997), pp. 3840-3845.

[10] R. Dubessy, T. Liennard, P. Pedri, and H. Perrin, Critical rotation of an annular superfluid bose-einstein condensate, Phys. Rev. A, 86 (2012), p. 011602.

[11] C. Elphick And E. Meron, Localized structures in surface waves, Physical Review A, 40 (1989), p. 3226 .

[12] _ Comment on "Solitary waves generated by subcritical instabilities in dissipative systems", Physical review letters, 65 (1990), p. 2476.

[13] S. Fauve and O. Thual, Solitary waves generated by subcritical instabilities in dissipative systems, Physical review letters, 64 (1990), p. 282.

[14] J. García-Ripoll and V. PÉRez-García, Vortex nucleation and hysteresis phenomena in rotating bose-einstein condensates, Phys. Rev. A, 63 (2001), p. 041603.

[15] A. Griffin, T. Nikuni, And E. Zaremba, Bose-condensed gases at finite temperatures, Cambridge University Press, 2009.

[16] P. C. Haluan, I. Coddington, P. Engels, and E. A. Cornell, Driving bose-einstein-condensate vorticity with a rotating normal cloud, Phys. Rev. Lett., 87 (2001), p. 210403.

[17] E. Hodby, G. Hechenblaikner, S. A. Hopkins, O. M. Maragò, and C. J. Foot, Vortex nucleation in bose-einstein condensates in an oblate, purely magnetic potential, Phys. Rev. Lett., 88 (2001), p. 010405.

[18] T. Isoshima And K. Machida, Instability of the nonvortex state toward a quantized vortex in a boseeinstein condensate under external rotation, Phys. Rev. A, 60 (1999), pp. 3313-3316.

[19] B. Jackson, N. Proukakis, C. Barenghi, and E. Zaremba, Finite-temperature vortex dynamics in Bose-Einstein condensates, Physical Review A, 79 (2009), p. 053615.

[20] Y. Kivshar and B. Malomed, Dynamics of solitons in nearly integrable systems, Reviews of Modern Physics, 61 (1989), p. 763.

[21] M. Krämer, L. Pitaevskit, S. Stringari, And F. Zambelli, Vortex nucleation and quadrupole deformation of a rotating bose-einstein condensate, Laser Physics, 12 (2002), pp. 113-120. 
[22] K. W. Madison, F. Chevy, V. Bretin, and J. Dalibard, Stationary states of a rotating boseeinstein condensate: Routes to vortex nucleation, Phys. Rev. Lett., 86 (2001), pp. 4443-4446.

[23] P. Mandel And T. ERneux, The slow passage through a steady bifurcation: delay and memory effects, Journal of statistical physics, 48 (1987), pp. 1059-1070.

[24] S. McEndoo And T. Busch, Small numbers of vortices in anisotropic traps, Physical Review A, 79 (2009), p. 053616.

[25] R. Navarro, R. Carretero-González, P. Torres, P. Kevrekidis, D. Frantzeskakis, M. Ray, E. Altuntaş, And D. Hall, Dynamics of a few corotating vortices in bose-einstein condensates, Phys. Rev. Lett., 110 (2013), p. 225301.

[26] A. Penckwitt, R. Ballagh, and C. Gardiner, Nucleation, growth, and stabilization of BoseEinstein condensate vortex lattices, Physical Review Letters, 89 (2002), p. 260402.

[27] C. Pethick and H. Smith, Bose-Einstein condensation in dilute gases, Cambridge University Press, 2002.

[28] L. Pismen, Vortices in Nonlinear Fields, Clarendon, UK, 1999.

[29] L. Pitaevskil, Phenomenological theory of superfluidity near the $\lambda$ point, Zh. Eksp. Teor. Fiz, 35 (1958), pp. 408-415.

[30] L. Pitaevskit and S. Stringari, Bose-Einstein condensation, Oxford University Press, 2003.

[31] N. Proukakis, S. Gardiner, M. Davis, and M. Szymanska, Quantum Gases: Finite Temperature and Non-Equilibrium Dynamics, Imperial College Press, London, 2013.

[32] N. Proukakis And B. Jackson, Finite-temperature models of Bose-Einstein condensation, Journal of Physics B: Atomic, Molecular and Optical Physics, 41 (2008), p. 203002.

[33] Z. Rapti, P. Kevrekidis, D. Frantzeskakis, and B. Malomed, On the modulational instability of the nonlinear Schrödinger equation with dissipation, Physica Scripta, 2004 (2004), p. 74.

[34] A. Recati, F. Zambelli, and S. Stringari, Overcritical rotation of a trapped bose-einstein condensate, Phys. Rev. Lett., 86 (2001), pp. 377-380.

[35] M. Rechtsman, J. Zeuner, Y. Plotnik, Y. Lumer, D. Podolsky, F. Dreisow, S. Nolte, M. SEgev, And A. Szameit, Strong transmission and reflection of edge modes in bounded photonic graphene, Nature, 496 (2013), pp. 196-200.

[36] H. Segur, D. Henderson, J. Carter, H. J., C.-M. Li, D. Pheiff, and K. Socha, Stabilizing the benjamin-feir instability, Journal of Fluid Mechanics, 539 (2005), pp. 229-271.

[37] T. P. Simula, S. M. M. Virtanen, And M. M. SalomaA, Surface modes and vortex formation in dilute bose-einstein condensates at finite temperatures, Phys. Rev. A, 66 (2002), p. 035601.

[38] S. Sinha And Y. Castin, Dynamic instability of a rotating bose-einstein condensate, Phys. Rev. Lett., 87 (2001), p. 190402.

[39] M. Tsubota, K. Kasamatsu, and M. Ueda, Vortex lattice formation in a rotating bose-einstein condensate, Phys. Rev. A, 65 (2002), p. 023603.

[40] E. Weinan, Dynamics of vortices in ginzburg-landau theories with applications to superconductivity, Physica D: Nonlinear Phenomena, 77 (1994), pp. 383-404.

[41] D. Yan, R. Carretero-González, D. Frantzeskakis, P. Kevrekidis, N. Proukakis, and D. SPIRN, Exploring vortex dynamics in the presence of dissipation: analytical and numerical results, Physical Review A, 89 (2014), p. 043613. 